\title{
Research on Inventory Strategy of Perishable Goods Considering Emergency Demand
}

\author{
Xiuxiu Li, Yuquan Cui \\ School of Mathematics, Shandong University, Jinan, China \\ *Corresponding author
}

\begin{abstract}
The research object of this paper was a single perishable product that does not immediately deteriorate. The emergency demand for the perishable goods was rarely studied, but it was practically significant. This article firstly used the piece wise exponential metamorphism function to characterize the non-immediate metamorphism. This paper also considered the emergency demand ignored previously. Then this paper explored the retailer's inventory strategy after introducing the emergency demand influence factor into the inventory level function and demand function. Finally, the impact of emergency demand factors on the retailer's single inventory strategy was compared, analyzed and expanded. Therefore, the practical significance of this paper is proved in theory.
\end{abstract}

Keywords: Non-immediate deterioration, The emergency demand, The inventory strategy

\section{INTRODUCTION}

With the progress of science and technology, people's requirements on products' quality are higher and higher. However, the deterioration and loss of products will inevitably occur in each process of the supply chain. As known, most fresh agricultural products are perishable. From the perspective of retailers, considering the inventory control of perishable products in the sales cycle will be conducive to improving the accuracy of retailers' demand prediction and promoting the development of fresh agricultural products industry chain. Recently, many scholars have studied and developed the replenishment strategy of perishable goods [1-4].

The research on perishable products began in 1977. In literature [1], it was assumed that the product was in exponential decay form and the deterioration coefficient was constant. However, the deterioration rate of perishable goods is not constant in real life. Scholars at home and abroad [5-7] introduced the concept of non-immediate metamorphism, but there are not many studies on the classification of the metamorphism rate of perishable products by stages, which is exactly the point explored in this paper. In addition, in the aspect of inventory control, most literature [8-11] considered the factors affecting the demand rate of perishable goods such as inventory level, freshness, price discount and the delay of stock shortage. But there are few studies on the factors affecting emergency demand. Therefore, this paper makes the following improvements and innovations based on the previous research work of scholars [10-15].

\section{PROBLEM DESCRIPTION AND ASSUMPTIONS}

This paper mainly studies the inventory system of the retailer who sells a non-immediate perishable product and the sales cycle of is $[0, T]$ divided into four stages. And in different stages of non-immediate deterioration, the impact coefficient of inventory level on the demand rate $\theta_{i}$ is different. The following assumptions are made.

(1) The retailer's order for other products for sale is relatively independent of the specific non-immediate spoiled perishable products studied in this paper.

(2) The inventory has no upper limit.

(3) The metamorphism of the non-immediate metamorphism is divided into two stages, and the decay form is exponential form.

(4) The system allows the shortage of goods to be delayed. But the delayed goods must to be delivered before the beginning of the next cycle. Referring to Abad[11], the delay rate of goods in shortage is expressed as $B(t)=e^{-K(T-t)}$ while $K \geq 0$ indicating the resistance after the delay of goods in shortage and $T-t$ the waiting time of customers in a single cycle. 


\section{ESTABLISHMENT OF MODEL}

\subsection{The Model Not Considering Emergency Demand}

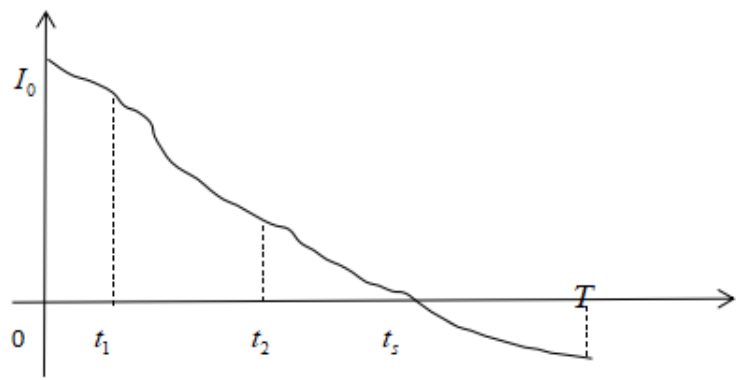

Figure 1 Schematic diagram of inventory level change curve

Figure 1 shows a simplified inventory level for the perishable product sales cycle $[0, T]$. The initial inventory of the sales cycle is $I_{0}$. The first stage is $\left[\begin{array}{ll}0, & t_{1}\end{array}\right]$ where the metamorphism coefficient is $\theta_{1}$. The second stage without price discount is $\left[t_{1}, t_{2}\right]$ while the coefficient is $\theta_{2}$. The second stage with price discount is $\left[t_{2}, t_{3}\right]$ and the coefficient is still $\theta_{2} .\left[t_{s}, T\right]$ is the out-of-stock phase allowed by the system and the demand rate is no longer affected by the inventory level. Therefore, the demand rate function in the sales cycle $[0, T]$ can be described as the following.

$$
\begin{cases}D_{0}+\left(\theta_{1}+\alpha\right) I(t) & 0 \leq t \leq t_{1} \\ D_{0}+\left(\theta_{2}+\alpha\right) I(t) & t_{1} \leq t \leq t_{2} \\ D_{0}+\left(\theta_{2}+\alpha\right) I(t)+\lambda & t_{2} \leq t \leq t_{s} \\ D_{0} B(t) & t_{s} \leq t \leq T\end{cases}
$$$$
D(\mathrm{t})=
$$

Then, the inventory system of the perishable product can be characterized by the following.

$$
\left\{\begin{array}{cc}
-D_{0}-\left(\theta_{1}+\alpha\right) I(t) & 0 \leq t \leq t_{1} \\
-D_{0}-\left(\theta_{2}+\alpha\right) I(t) & t_{1} \leq t \leq t_{2} \\
-D_{0}-\left(\theta_{2}+\alpha\right) I(t)-\lambda & t_{2} \leq t \leq t_{s} \\
-D_{0} B(t) & t_{s} \leq t \leq T
\end{array}\right.
$$

And the boundary condition is $I(0)=I_{0}, I\left(t_{1}\right)=I_{1}$, $I\left(t_{s}\right)=0$.

Solving Eq.2, it can be obtained that Eq.3. Also, $I_{1}\left(t_{1}\right)=I_{2}\left(t_{1}\right)$ and $I_{2}\left(t_{2}\right)=I_{3}\left(t_{2}\right)$ due to the continuity of $I(t)$. Therefore, we can get

$$
\begin{aligned}
& \left\{\begin{array}{l}
I_{1}=-D_{0}\left(1-e^{-\left(\theta_{1}+\alpha\right) t}\right) /\left(\theta_{1}+\alpha\right)+e^{-\left(\theta_{1}+\alpha\right) t} I_{0} \\
I_{2}=I_{1} e^{-\left(\theta_{1}+\alpha\right)\left(t_{1}-t\right)} \\
I_{3}=\frac{\lambda+D_{0}}{\theta_{2}+\alpha}\left[e^{-\left(\theta_{1}+\alpha\right)\left(t_{1}-t\right)}-1\right] \\
I_{4}=\frac{D_{0} e^{-K T}}{K}\left[e^{K t_{s}}-e^{K t}\right] \\
I_{0}=\frac{\lambda+D_{0}}{\theta_{2}+\alpha} \cdot\left[e^{\left(\theta_{2}+\alpha\right)\left(t_{s}-t_{2}\right)}-e^{\left(\theta_{2}+\alpha\right)\left(t_{2}-t_{1}\right)}\right] \cdot e^{\left(\theta_{1}+\alpha\right) t_{1}} \\
+\frac{D_{0} \cdot e^{\left(\theta_{1}+\alpha\right) t_{1}}}{\theta_{1}+\alpha} \cdot\left[1-e^{-\left(\theta_{1}+\alpha\right) t_{1}}\right]
\end{array}\right.
\end{aligned}
$$

$$
\begin{aligned}
& I_{1}= \\
& \frac{\lambda+D_{0}}{\theta_{2}+\alpha} \cdot\left[e^{\left(\theta_{2}+\alpha\right)\left(t_{s}-t_{2}\right)}-1\right] \cdot e^{\left(\theta_{2}+\alpha\right)\left(t_{2}-t_{1}\right)} .
\end{aligned}
$$

For the convenience of calculation, the following paper still use $I_{0}$ and $I_{1}$ to express the more complex formula. Based on the above calculation, the following proposition can be obtained.

Proposition 1: The maximum deferred delivery quantity within $[0, T]$ is $S=\frac{D_{0} e^{-K T}}{K}\left(e^{K T}-e^{K t_{s}}\right)$, and the maximum order quantity is $Q=I(0)+S=I_{0}+\frac{D_{0} e^{-K T}}{K} \cdot\left(e^{K T}-e^{K t_{s}}\right)$. There is a discussion of the retailer's cost. Let $P C$ be the purchase and ordering costs, including fixed costs, then $P C=A_{0}+w Q$. Let $S R$ be the sales revenue, then $S R=p \int_{0}^{T} D(t) d t$ where $p$ is the selling price per unit. Let $H C$ be the total inventory holding expense. Specifically, the inventory holding cost per unit is the time-varying function $C_{h}(t)$ and it increases with the increase of storage time, i.e. $d C_{h}(t) / d t \geq 0$. Then $H C=\int_{0}^{t_{1}} C_{h}(t) I_{1} d t+\int_{t_{1}}^{t_{2}} C_{h}(t) I_{2} d t+\int_{t_{2}}^{t_{s}} C_{h}(t) I_{3} d t$.

There is a certain out-of-stock cost in the inventory system. Let $C_{s}$ be the cost of shortage and $S C$ be the total cost of shortage.

Then

$S C=C_{s} \int_{t_{s}}^{T}-I_{4}(t) d t=C_{s} \int_{t_{s}}^{T} \frac{D_{0} e^{-K T}}{K}\left(e^{K t}-e^{K t_{s}}\right) d t$.

For the quantity of shortage during the period of shortage, 
there is the opportunity cost caused by unit sales loss. Let $C_{0}$ be the unit opportunity cost and $O C$ be the total opportunity cost of the system. Then

$O C=C_{0} \int_{t_{s}}^{T} \alpha\left(1-e^{-K(T-t)}\right) d t=\alpha C_{0}\left[\left(T-t_{s}\right)-\frac{1}{K}+\frac{e^{K t_{s}-K T}}{K}\right]$.

Then the total profit of the retailer is $T P=S R-(P C+H C+S C+O C)$.

By analyzing the profit function of the above retailers, the following proposition can be obtained.

Proposition 2: On the premise of the above hypothesis, the retailer has the optimal starting discount point $t_{2}^{*}$ in the sales

cycle $[0, T]$ so that $\left.\frac{\partial T P}{\partial t_{2}}\right|_{t_{2}=t_{2}^{*}}=0$.

Proof: Firstly, the continuity of $T P$ is known and the differentiability of $T P$ with respect to $t_{2}$ can be obtained from its expression.

$$
\begin{aligned}
& \frac{\partial^{2} T P}{\partial t_{2}{ }^{2}}= \\
& p\left(\lambda+D_{0}\right)\left[-e^{t_{s}-t_{2}}-e^{t_{2}-t_{1}}-\left(\theta_{2}+\alpha\right) e^{\left(\theta_{2}+\alpha\right)\left(t_{s}-t_{2}\right)}\right. \\
& \left.+\left(\theta_{2}+\alpha\right)\left(1-e^{-\left(\theta_{1}+\alpha\right) t_{1}}\right) \cdot e^{\left(\theta_{2}+\alpha\right)\left(t_{2}-t_{1}\right)+\left(\theta_{1}+\alpha\right) t_{1}}\right] .
\end{aligned}
$$

It can be judged that the second partial derivative is less than zero. Then the profit function is convex with expect to $t_{2}$. So there is an optimal discount starting time $t_{2}^{*}$, and it satisfies $\left.\frac{\partial T P}{\partial t_{2}}\right|_{t_{2}=t_{2}^{*}}=0$.

\subsection{The Model Considering Emergency Demand}

Firstly, consider that the emergency demand occurs in the first stage of the metamorphism period. The influence factor of demand rate is $\beta_{1}$ and the demand rate function can be obtained. Correspondingly, the amount of inventory per unit time is partially changed.

$$
\frac{d I(t)}{d t}=\left\{\begin{array}{ll}
-D_{0}-\left(\theta_{1}+\alpha\right) I(t)-\beta_{1} & 0 \leq t \leq t_{1} \\
-D_{0}-\left(\theta_{2}+\alpha\right) I(t) & t_{1}<t \leq t_{2} \\
-D_{0}-\left(\theta_{2}+\alpha\right) I(t)-\lambda & t_{2}<t \leq t_{s} \\
-D_{0} B(t) & t_{s}<t \leq T
\end{array} .\right.
$$

Similar to the above analysis and discussion when emergency demand not considered, the phased inventory level during the sales period can be obtained as follows.

$$
\left\{\begin{array}{l}
I_{1}=-D_{0}\left(1-e^{-\left(\theta_{1}+\alpha\right) t}\right) /\left(\theta_{1}+\alpha\right)+e^{-\left(\theta_{1}+\alpha\right) t} I_{0}^{1} \\
I_{2}=I_{1}^{1} e^{-\left(\theta_{1}+\alpha\right)\left(t_{1}-t\right)} \\
I_{3}=\frac{\lambda+D_{0}}{\theta_{2}+\alpha}\left[e^{-\left(\theta_{1}+\alpha\right)\left(t_{1}-t\right)}-1\right] \\
I_{4}=\frac{D_{0} e^{-K T}}{K}\left[e^{K t_{s}}-e^{K t}\right]
\end{array} .\right.
$$$$
I_{1}^{1}=\frac{\lambda+D_{0}}{\theta_{2}+\alpha} \cdot\left[e^{\left(\theta_{2}+\alpha\right)\left(t_{s}-t_{2}\right)}-1\right] \cdot e^{\left(\theta_{2}+\alpha\right)\left(t_{2}-t_{1}\right)},
$$

$I_{0}^{1}=$

$$
\begin{aligned}
& \frac{\lambda+D_{0}}{\theta_{2}+\alpha} \cdot\left[e^{\left(\theta_{2}+\alpha\right)\left(t_{s}-t_{2}\right)}-e^{\left(\theta_{2}+\alpha\right)\left(t_{2}-t_{1}\right)}\right] \cdot e^{\left(\theta_{1}+\alpha\right) t_{1}} \\
& +\frac{\left(D_{0}+\beta_{1}\right) \cdot e^{\left(\theta_{1}+\alpha\right) t_{1}}}{\theta_{1}+\alpha} \cdot\left[1-e^{-\left(\theta_{1}+\alpha\right) t_{1}}\right] .
\end{aligned}
$$

It can be seen that considering emergency demand occurs in the first stage of the metamorphism stage, the impact on $I(t)$ is mainly reflected in the first stage. However, the introduction of emergency demand has an impact on the overall profit of the supply chain beyond the first stage.

Proposition 3: In the case that emergency demand occurs in the first stage of the metamorphism period, when $w<\left(1-e^{-\left(\theta_{1}+\alpha\right) t_{1}}\right) \cdot \int_{0}^{t_{1}} C_{h}(t) d t$, the difference value of sales revenue of the retailer in the sales cycle $[0, T]$ increases with the increase of the influence factor $\beta_{1}$.

Proof: Similar to the above discussion, $T P^{1}=S R^{1}-\left(P C^{1}+H C^{1}+S C+O C\right)$ is the new sales revenue. Let $\triangle T P=T P^{1}-T P$ be the change in sales revenue. Similarly, the difference of kinds of costs can be presented such as $\triangle P C=P C^{1}-P C$. Then $\Delta T P=\Delta S R-\triangle P C-\triangle H C$.

In order to explore the relationship between the change in sales revenue and the impact factor of emergency demand $\beta_{1}$

then,

$$
\begin{aligned}
& \frac{\partial \Delta T P}{\partial \beta_{1}}=t_{1}- \\
& \frac{e^{\left(\theta_{1}+\alpha\right)}\left(1-e^{-\left(\theta_{1}+\alpha\right) t_{1}}\right)\left[w-\left(1-e^{-\left(\theta_{1}+\alpha\right) t_{1}}\right)^{2} \cdot \int_{0}^{t_{1}} C_{h}(t) d t\right]}{\theta_{1}+\alpha} .
\end{aligned}
$$




$$
\begin{aligned}
& \text { As can be seen, } \frac{\partial \Delta T P}{\partial \beta_{1}}>0 \quad \text { if } \\
& w<\left(1-e^{-\left(\theta_{1}+\alpha\right) t_{1}}\right) \cdot \int_{0}^{t_{1}} C_{h}(t) d t .
\end{aligned}
$$

That is the difference value of sales revenue of the retailer in the sales cycle $[0, T], \Delta T P$ increases with the increase of $\beta_{1}$. In other words, the greater the impact factor of emergency demand $\beta_{1}$ is, the greater the profit difference value between the supply chain of the two models $\triangle T P$ is. It is also consistent with real life. Therefore, it is necessary to consider the emergency demand. In addition, the introduction of the impact factor of emergency demand $\beta_{1}$ increases the maximum the order quantity of retailers. Specifically, the impact of inventory control on retailers can be seen from the following.

Proposition 4: In the sales cycle $[0, T]$, considering the emergency impact factor $\beta_{1}$, the maximum delayed delivery quantity is $S=\frac{D_{0} e^{-K T}}{K}\left(e^{K T}-e^{K t_{s}}\right)$, and the maximum order quantity is $Q=I(0)+S=I_{0}^{1}+\frac{D_{0} e^{-K T}}{K} \cdot\left(e^{K T}-e^{K t_{s}}\right)$ where $I_{0}^{1}$ is given by the Eq.9.

In reality, the occurrence time of emergency demand may not be able to strictly defined in the specific metamorphism stage. So the logic function is introduced. $l_{i}=\left\{\begin{array}{c}0, \text { Emergency demand do not occurs at i } . \\ 1, \text { Emergency demand occurs at i. }\end{array}\right.$ Meantime, let $\beta_{1} \beta_{2}$, and $\beta_{3}$ be the emergency impact factors in different metamorphism stages. Therefore, the demand rate function $D(t)$ and $d I(t) / d t$ is partially changed. Combined with the boundary conditions, the phased expression of $I(t)$ can be obtained.

$$
\begin{aligned}
& \frac{d I(t)}{d t}=\left\{\begin{array}{l}
-D_{0}-\left(\theta_{1}+\alpha\right) I(t)-l_{1} \beta_{1} \\
-D_{0}-\left(\theta_{2}+\alpha\right) I(t)-l_{2} \beta_{2} \\
-D_{0}-\left(\theta_{2}+\alpha\right) I(t)-\lambda-l_{3} \beta_{3} \\
-D_{0} B(t)
\end{array}\right. \\
& \left\{\begin{array}{l}
I_{1}=-\left(D_{0}+l_{1} \beta_{1}\right)\left(1-e^{-\left(\theta_{1}+\alpha\right) t}\right) /\left(\theta_{1}+\alpha\right)+e^{-\left(\theta_{1}+\alpha\right) t} I_{0}^{2} \\
I_{2}=I_{1}^{2} e^{-\left(\theta_{1}+\alpha\right)\left(t_{1}-t\right)} \\
I_{3}=\frac{\lambda+D_{0}+l_{2} \beta_{2}}{\theta_{2}+\alpha}\left[e^{-\left(\theta_{1}+\alpha\right)\left(t_{1}-t\right)}-1\right] \\
I_{4}=\frac{D_{0} e^{-K T}}{K}\left[e^{K t_{s}}-e^{K t}\right]
\end{array}\right.
\end{aligned}
$$

Meantime, since the continuity of $I(t)$, it can be obtained that $I_{1}^{2}\left(t_{1}\right)=I_{2}^{2}\left(t_{1}\right)$ and $I_{2}^{2}\left(t_{2}\right)=I_{3}^{2}\left(t_{2}\right)$. Similar to the case of emergency demand factors in the first stage of the metamorphism stage mentioned above, the same discussion can be carried out.

\section{CONCLUSIONS AND PROSPECTS}

By assuming the consumption of items modification treatment costs, the cost of maintaining inventory cost items, the discount coefficient and so on, this paper built the metamorphic immediately inventory model. Then, the inventory strategy was explored. It is worth mentioning that this article used the piece wise exponential metamorphism function to characterize the non-immediate metamorphism. In addition, this paper considered the emergency demand. The conclusions also proved its practical significance.

However, in the second stage of the non-immediate deterioration period, the linear addition hypothesis is adopted for the effect of retailer discount rate on demand rate and the effect of emergency demand factor on demand rate in different stages. But in real life, there may be other more complex forms of influence, which can be further analyzed and extended. And it is more suitable to apply time-varying effects in reality.

\section{REFERENCES}

[1] COHEN M A, Joint pricing and ordering policy for exponentially decaying with known demand, J. Naval Research Logistics Quarterly. 1977,24(2):257-268.

[2] P.L.Abad, Optimal pricing and lot-sizing under conditions of perishability and partial backordering, $\mathrm{J}$. Management Science. 1996, Vol.42 (8), pp.1093-1104.

[3] MIN J, ZHOU Y, Inventory model for non-instantaneous deteriorating items under stock-dependent selling rate, J. Journal of Systems Engineering. 2009,24(2):198-204.

[4] Xu lijian, Pang Qinghua, Optimal ordering strategy of spoiled goods with price discount, J. Journal of Qingdao University (natural science edition). 2001(03):27-33.

[5] George C.Philip, A Generalized EOQ Model for Items with Weibull Distribution Deterioration.IIE Transactions. 1974, Vol.6 (2), pp.159-162.

[6] Richard P.Covert, George C.Philip, A EOQ Model for Items with Weibull Distribution Deterioration.IIE Transactions. 1973, Vol.5 (4), pp.323-326.

[7] Kun-ShanWu, Liang-Yuh Ouyang, Chih-Te Yang, An optimal replenishment policy for non-instantaneous 
deteriorating items with stock-dependent demand and partial backlogging, J. International Journal of Production Economics. 2006,101(2).

[8] Ai Xue-yi, Zhang Jin-long, Xu Hao-xuan, Wang Lin, Joint replenishment optimization of non-immediate deteriorated goods with demand dependent on deterioration time, J. Control and decision. 2008,33(04):671-678.

[9] PI Xing, Meng Weidong, Huang Bo, Jiang Jihai, A VMI model of deterioration products related to price discount and quantity delay rate of shortage, J. Industrial engineering and management. 2010,15(01):21-24+36.

[10] LI Gui-ping, DUAN Yong-rui, HUO Jia-zhen, XIONG De-ping, Inventory Policy for Non-Instantaneously Deteriorating Items with Time-varying Holding Cost and Deterioration Rate, J. Chinese Journal of Management Science. 2015,23(08):122-131.

[11] Zhang Chuan, Pan Dehui, Optimal replenishment strategy of perishable goods with random demand deterioration rate in distribution system like Weibull distribution, J. Theory and practice of systems engineering. 2008(09):87-91.

[12] Liu Mingwu, Wei Xiaomei, Inventory allocation strategy of shuangyuan supply under two types of demand, J. Systems engineering. 2016,34(04):84-88.

[13] Cao Yu, Yi Chaoqun, Wan Guangyu, Dynamic pricing, service level and production control strategy of random production inventory model for non-immediate deterioration, J. Theory and practice of systems engineering. 2018(38):1717-1731.

[14] Ke Liu, Jing-An Li, Kin Keung Lai, Single period, single product newsvendor model with random supply shock, J. European Journal of Operational Research. 2004 (158) 609-625.

[15] Luqi Wang, Zhijian CHEN, Mingyao CHEN, Ruijie ZHANG, Inventory Policy for a Deteriorating Item with Time-Varying Demand Under Trade Credit and Inflation, J. Journal of Systems Science and Information. 2019(07) 115-133. 